\title{
A method for micrometer resolution patterning of primary culture neurons for SPM analysis
}

P. Degenaar ${ }^{1}$, B. Le Pioufle ${ }^{3}$, L. Griscom, A. Tixier ${ }^{2}$, Y. Murakami ${ }^{1}$, K. Yokoyama ${ }^{1}$, E. Tamiya $^{1}$, H. Fujita ${ }^{2}$

1 The School of Materials Science, Japan Advanced Institute of Science and Technology, Hokuriku, 1-1 Asahidai, Tatsunokuchi, Ishikawa 923-1292 Japan

2 LIMMS / CNRS Institute of Industrial Science, University of Tokyo,7-22-1 Roppongi, Minato-ku,

Tokyo 106-8558, Japan

${ }^{3}$ Ecole Normale Supérieure de Cachan, Campus de Ker-Lann, 35170 Bruz, France

\section{Corresponding author:}

P Degenaar

The School of Materials Science,

Japan Advanced Institute of Science and Technology, Hokuriku,

1-1 Asahidai,

Tatsunokuchi,

Nomi-gun,

Ishikawa

923-1292

Japan

Te: 0761511663

Fax 0761511665

Email: p.degenaar@physics.org

Topic: Biotechnology 


\section{SUMMARY}

In this work we present a method for ultra-fine patterning of primary culture neuron cell growth, which is compatible for scanning near-field optical atomic force microscopy (SNOAM) analysis. SNOAM uses near field optics to break the fundamental diffraction limit imposed on normal microscopy. SNOAM can achieve sub-100nm optical resolutions, but requires transparent, open substrates. The ability to do physiological measurements on patterns of neurons, combined with ultra high resolution optical and fluorescent analysis is useful to the study of long term potentiation. The patterning method consists of chemical guidance with an element of physical confinement and allows for ultra fine patterning of neural growth on transparent glass substrates. Substrates consist of microfabricated perfluoropolymer barrier structures on glass. Poly-1-lysine was selectively deposited using a silicone based microfluidic stencil aligned to the perfluoropolymer/glass substrate. Primary culture neurons were extracted from 8 day old chicks and grown for 3 days to form good networks. This patterning system shows very specific growth with patterning separations down to the level of individual neurites. Fluorescent imaging was carried out on both cell viability during growth and immuno-tagged microtubule associated proteins on the neurites. Neurons inside the patterned structures were imaged and analyzed with a tapping mode SNOAM. 
Long term potentiation (LTP) of synaptic transmission in the hippocampus is the primary experimental model for investigating the synaptic basis of learning and memory in vertebrates ${ }^{1}$. LTP is believed to be linked to the glutamate cycle ${ }^{2}$ and activation of amino acid receptors such as the N-methyl-D-aspartate receptor complex ${ }^{3}$. Studies in this area have involved electrophysiological study with patch clamping techniques and high-resolution fluorescence imaging with confocal microscopy. In our research we aim to improve this method with the introduction of two techniques; Patterning of neurons to the level of individual synapses and super high resolution Near-field optical microscopy $y^{4,5,6}$

Confocal microscopy is a major improvement over traditional focal microscopy techniques, but it is still limited by the diffraction limit of light in the lateral plane and by out of focus light in the vertical plane. Scanning near-field optical atomic-force microscopy (SNOAM, also known as NSOM and SNOM) breaks through the diffraction limit by using near-field optics. In SNOAM, a bent optical fiber with a sub-wavelength aperture at the tip is brought to within a few nanometers of the sample. The evanescent field around the tip aperture interacts with the sample to cause optical scattering or fluorescence stimulation. The sample is then scanned to build up an image. Optical resolutions between 50-100nm and topographical resolutions of a few hundreds of nanometers are presently possible, but this will improve as the technology matures.

The patterned growth of in vitro neurons is a key feature in the development of both fundamental and applied research in the neuroscience field. Moreover patterning of neurons, whereby individual neurites are guided, will allow for better studies of neuron connectivity, synapse formation and studies into long term potentiation (LTP). 
Neuron guidance can be carried out through physical, chemical or electrical cues ${ }^{7,8}$. A number of studies for the patterning of cell lines such as PC12 and BCE cell lines has already been done ${ }^{9}$. However primary cell lines have proven to be more difficult to pattern. Some microfluidic systems have shown to be capable of patterning primary culture cells ${ }^{10,11}$. However for scanning probe microscopy, relatively flat and open systems are required. SNOAM samples with pattern roughness of more than a few microns seriously degrades imaging capability as will be discussed later. Thus we have used chemical patterning cues with limited topographic confinement on transparent glass substrates for SNOAM analysis. Samples for SNOAM analysis also need to be transparent for optimum optical detection. So we have fabricated chips with transparent topographical structures of amorphous perfluoropolymer on glass substrates.

To this end, special growth substrates have been designed using microlithographic techniques, whereby topographic microstructures, and chemical patterning are formed on the same chip. Topographical patterns are formed by etching of amorphous perfluoropolymer on top of the glass substrates. It is believed that hydrophobic perfluoropolymers absorbs albumin proteins from the growth medium, which is repulsive to cells ${ }^{12}$. Chemical patterning of a poly-l-lysine growth matrix on the glass substrates has been carried out with microfluidic stencils. Poly-1-lysine growth matrix contains many amide groups which promote cell adhesion ${ }^{8}$. Thus this method creates structures which alternate between chemically attractive and repulsive regions.

In this study, topographical and chemical patterning are combined: first by fabrication of the topographical confinement using micro-lithography, and second, by 
patterning of the chemical adhesion molecules using a micro-stencil. Use of a micro stencil demonstrates a way to pattern molecules on surfaces where the topographical patterns are already formed. Using this method it is possible to create $10 \mu \mathrm{m}$ paths and achieve guidance of individual neurites. It is also desirable to integrate this patterning system with microelectrode arrays and to apply this technique to commercial chips such as the Med 64 system from Panasonic ${ }^{13}$.

\section{MATERIALS AND METHODS}

The experimental method involves the fabrication of the patterned glass/perfluoropolymer growth substrates and the silicone elastomer micro stencils, as can been seen in Fig. 1(a, b). Final preparation of the growth substrate is achieved by alignment and patterning of the adhesion molecules to the substrate using the stencil, as can been seen in Fig. 1(c). Finally the experimental protocols for cell growth and imaging are explained.

\section{Perfluoropolymer pattern fabrication}

Topographic patterns for neural guidance were fabricated using Cytop (Asahi Glass Co.) CTL-809M type amorphous perfluoropolymer, by photolithographic methods. The neuron path is made by etching the Cytop to give micrometer size trenches on a glass substrate ${ }^{14}$. Cytop displays highly hydrophobic properties and acts as a barrier to neuron growth.

The fabrication process is illustrated in Fig. 1(a). Initially Cytop is spin coated on cleaned glass slides. Spin coating at 1000rpm for 20 seconds, gives $2 \mu \mathrm{m}$. After spin coating, the sample is baked at $100^{\circ} \mathrm{C}$ for $30 \mathrm{~min}$. The thickness is adjusted with 
up to four successive spincoated layers. Next, 200nm of aluminum is evaporated onto the sample, which serves as an etch stop for Cytop patterning. Aluminum is photolithography etched to reveal the patterns, followed by reactive ion etching (RIE) of the Cytop layer until the glass is reached. RIE etches the Cytop at a rate of 200nm/min using an oxygen plasma in a parallel plate reactor. The gas flow rate is $100 \mathrm{sccm}$ (standard cubic centimeter per minute), with pressures of 50-70 mtorr, and a radio frequency power of 50W. In the final step of the etching process the aluminum is removed by aluminum etchant to reveal the hydrophobic areas of the Cytop layer. The samples are then washed and dried for preparation of the biological and chemical patterning. However it is noted that the original hydrophobicity of the Cytop coating was decreased after removal of the aluminum layer. The hydrophobic nature of the surface can be restored by curing the samples at above $100^{\circ} \mathrm{C}$.

\section{PDMS stencil fabrication}

The microfluidic stencil for patterning of chemicals and cell adhesion molecules are fabricated using polydimethylsiloxane, PDMS, elastomer (Sylgard RTV-184 supplied by Dow Corning Corp.). The stencil fabrication is illustrated in Fig. 1 (b). A lift-off molding technique is employed using innovative three-dimensional multitiered SU-8 thick negative-photoresist structures ${ }^{15}$. This allows the fabrication of complex lines and patterns for deposition of chemicals on a smooth substrate, while including multiple inlet pores in the PDMS membrane. Fabrication of multi-level structures by micromolding can be made through successive spincoating and exposure of multiple layers of SU-8 (Microchem Corp) negative photo resist. The first layer of SU-8 makes the patterns in the $\mathrm{x}-\mathrm{y}$ plane for deposition of chemicals. Which can be patterned in lines or a grid, with paths 10 to $50 \mu \mathrm{m}$ wide (The structures were $20-40$ 
$\mu \mathrm{m}$ tall) the second SU-8 layer is made so that an array of inlet pores (50 micron square and 150 microns deep) were formed connected with the horizontal patterns.

The PDMS polymer is mixed with hardener at 10:1 and degassed before molding. To insure that the inlet pores are completely pierced, the micro-mould is clamped to a poly-acrylic plate with a hole for injection of the PDMS. After injection, the whole system is cured for one hour at $60^{\circ} \mathrm{C}$.

The microfluidic samples are peeled off and aligned the glass/Cytop substrates. This system permitted multiple reuse of the SU- 8 micro mold. To aid in the insertion of liquid into the microfluidic channels the interior surfaces need to be rendered hydrophilic before use. The channels can be rendered selectively hydrophilic through one minute of $\mathrm{O}_{2}$ plasma treatment in a parallel plate RIE system.

\section{Alignment and deposition of the poly-l-lysine}

The final stage in the substrate preparation is illustrated in Fig. 1(c). The PDMS stencils are aligned to the patterned growth substrate using a manipulator arm and an inverted microscope. The PDMS stencils are placed upside-down on glass slides. A drop of water between the glass and the PDMS stencil prevents adhesion. The Cytop/glass substrate are inverted and attached to the manipulator arm. It is then lowered onto the PDMS, adjusting the manipulator arm for alignment. Using this technique, $1 \mu \mathrm{m}$ precision is possible, which is adequate for the $\geq 10 \mu \mathrm{m}$ patterns made by this method. An example of the alignment of the stencil to the patterned substrate can be seen in Fig. 2. 


\section{Recycling}

Cytop is inert to most chemicals, so samples can be recycled for re-use. The recycling involves washing thoroughly in water, followed by dipping in 5\% (v/v) bleach (Domestos) solution for about 5 - 10 minutes to remove the biological material. Further washing in soapy water, followed by thorough washing in milliQ (18.2 $\mathrm{M} \Omega$ ) water is then necessary so that no salt deposits occur on drying. The clean sample is annealed in a dry oven at $120^{\circ} \mathrm{C}$ for 1 hour to increase its hydrophobicity. The PDMS stencil is then re-aligned, as for the process above, and the poly-l-lysine is re-applied.

\section{Microfluidic patterning of adhesive proteins}

Poly-l-lysine is deposited by immersing the stencil masked glass/Cytop substrate in $2.5 \mathrm{mg} / \mathrm{ml}$ solution for 4 hours. The PDMS stencil masks the Cytop structures, thus the poly-l-lysine is deposited only in the glass channels. After 4 hours the sample is washed in distilled water and sterilized under UV in a clean bench for 30-minutes. The substrates are then put in petri dishes and filled with medium 30minutes prior to cell extraction.

\section{Dissociation and cell culture}

The neurons in this research are extracted from the frontal hemispheres of chick embryos. Eggs are incubated for 8 days in an egg incubator, sterilized in $70 \% \mathrm{v} / \mathrm{v}$ ethanol and entered into a clean bench. The egg is opened with a dissection scissors and the embryo taken out and put onto a warm dish of phosphate buffer solution (PBS). The chick is killed by decapitation and the frontal hemispheres of the brain are extracted. The hemispheres are chopped slightly and incubated in a test tube 
containing $1 \mathrm{ml}$ of trypsine solution in a water bath at $37^{\circ} \mathrm{C}$ for 30 minutes. After this time, the test tube is re-entered into the clean bench and the trypsine is removed by gentle washing with growth medium consisting of Dulbecco's modified eagles medium (DMEM), supplemented with $10 \%(\mathrm{v} / \mathrm{v})$ fetal bovine serum, $1 \%(\mathrm{v} / \mathrm{v})$ penicillin/streptomycin, 3.7g/L $\mathrm{NaHCO}_{3}, 0.2 \%$ (v/v), and 2mM L-glutamine. The cells are then gently dispersed into the solution using a pipette, which are softened using the clean bench Bunsen burner. Cells are counted via haemocytometer with typical yields in the region of $15 \times 10^{6}$ cells for the two hemispheres. Cell pattern concentrations used on the growth substrates are $5 \times 10^{5}$. Much higher concentrations lead to less specific growth, much less result in no growth. All neuron preparation are carried out under the sterile conditions of a clean bench. Growth is carried out in an incubator at $37^{\circ} \mathrm{C}$ and a $5 \% \mathrm{CO}_{2}$ atmosphere with saturated humidity.

\section{Staining with fluorescein diacetate}

$50 \mu \mathrm{M}$ solutions of fluorescein diacetate (FDA) (Molecular Probes) are made in DMSO. Then for experimentation $20 \mu \mathrm{l}$ of the $50 \mu \mathrm{M}$ FDA are diluted in $5 \mathrm{ml}$ of milliQ water. For tests $50 \mu 1$ of the diluted FDA is inserted into cell media containing $5 \mathrm{ml}$ of medium. Imaging is carried out within 5 minutes of FDA insertion.

\section{Tau protein antibody tagging}

Cells are initially fixed in $10 \%$ paraformaldehyde solution for $10 \mathrm{mins}$ and then gently washed with PBS. The cells are then blocked with 10\%(v/v) fetal bovine serum in PBS for 1 hour before insertion of the primary anti-tau antibodies (Sigma). The antibodies are used at a concentration of 1:100 from the commercial aliquot. After a further 8 hours the samples are washed in PBS and blocked again for 1 hour. The anti- 
tau antibodies have rabbit affinity, thus the secondary antibodies were anti-rabbit Alexa-fluor 488 (Molecular Probes).

Imaging is carried out on an Olympus IX70 inverted microscope with a Yokogawa CSU10 laser-scanning unit. Optical stimulation is carried out with a 488nm laser line from an argon-ion laser.

\section{NMDA receptor tagging}

Cells are initially fixed in $10 \%$ paraformaldehyde solution for $10 \mathrm{mins}$ and then gently washed with PBS. The cells are then blocked with 10\%(v/v) fetal bovine serum in PBS for 1 hour before insertion of the primary anti-NMDA-NR1 antibodies (Sigma). The antibodies are used at a concentration of 1:2000 from the commercial aliquot. After a further 8 hours, the samples are washed in PBS and blocked again for 1 hour. The anti-NMDA-NR1 antibodies have rabbit affinity, thus secondary antibodies are anti-rabbit FITC (Molecular probes).

\section{SNOAM}

Topographic and near-field fluorescent analysis is carried out with a Seiko SPA700 series SNOAM. The SPA700 SNOAM uses bent optical fibers, which operate in tapping mode. For imaging, the probe tip is vibrated several nanometers above the sample surface. An evanescent near field, which forms around the aperture of the tip, interacts with the optically scattering material of the sample or stimulates fluorescent probes. The sample is then scanned up to $72 \mu \mathrm{m}^{2}$ to build up either an optical scattering or fluorescence emission image. Topographic images are taken simultaneously with the optical images. A diagram of the SNOAM system can be seen in Fig. 3. 
The probe sample distance is maintained by an atomic force feedback mechanism. More precisely the probe is operated in differential mode feedback. The optical fibre is mounted on a bimorph and vibrated vertically against the specimen stage at its resonant frequency. Atomic forces between the probe and the sample cause shifts in the resonant frequency, which can be detected for the feedback loop. The vibration amplitude is monitored by detecting a laser beam, which is reflected off a mirrored surface on the optical fibre cantilever. Feedback electronics are controlled by a commercial AFM controller (model SPI 3800, Seiko Instruments Inc). Differential force feedback has many advantages over contact force and shear force feedback mechanisms as it cuts down on destructive lateral forces on the cell membrane.

Laser stimulation is carried out with a $488 \mathrm{~nm}$ laser line from a multiline aircooled argon-ion laser. The laser intensity of the $488 \mathrm{~nm}$ laser line is a few milliwatts to prevent overheating of the tip aperture. The throughput efficiency of the tip depends on the size of the tip aperture, but is typically $10^{-5}$ for a $100 \mathrm{~nm}^{\mathrm{n}} \mathrm{ip}^{5}$. Signal light from the sample is corrected via objective lens (40x and 100x oil immersion type) and separated by dichroic mirror to the CCD camera and detectors. A CCD camera and a photomultiplier are used respectively for probe positioning and signal detection.

Probes are bought from Seiko Instruments Inc. They are produced from 125_m optical fibers $^{16}$, which are chemically etched to make a tip, bent by $\mathrm{CO}_{2}$ irradiation, and coated in 100-200nm of evaporated Aluminum. Apertures were drilled with a focused ion beam. Spring constants of the probes have been calculated theoretically to be $2-20 \mathrm{~N} / \mathrm{m}$. The probes resonance quality is determined by their Q factor, which is between 200-500. 


\section{RESULTS}

Cell patterning was very successful with capabilities of patterning down to the level of individual neurites. Over large areas patterning was specific with only occasional cases of non-guided growth.

Tests were done to see the effect of poly-1-lysine on the Cytop/glass patterns without using the stencil. When substrates were dipped for long periods of time, the growth was non-specific. If the samples were dipped in the poly-lysine for 2-3 minutes there would be preferential growth in the glass channels, but still some nonspecific growth on the Cytop. Very short or no poly-1-lysine deposition times led to no growth. This indicates that poly-1-lysine adheres to the glass faster than to the Cytop.

In our experiments cells formed optimum networks at 72 hours of cultivation on the substrates. After this time, growth continued, but the condition of the cells decreased. Generally 1 week is set as the useful lifetime of the cells. The cell condition is closely related to the condition of the growth substrate. It is known that poly-l-lysine desorbs from the surface over time during growth ${ }^{17}$. Desorption can be seen in the form of axons which begin to float. In this research 72 hours is sufficient to form good networks. For longer-term studies into LTP this lifespan needs to be extended. At high concentrations cells emit their own growth substrates. In tests, when cell concentrations are higher than $1 \times 10^{6}$ cells $/ \mathrm{ml}$, cells will grow on most substrates for extended periods of time. However, at that concentration, neurons form a very dense layer and it is not possible to effectively guide neurons with chemical cues. It is however possible to use different methods of depositing amide groups 
and/or other functional groups ${ }^{8,18}$ on the glass surface with our method, so this is not a fundamental problem.

The total concentration of cells was $5 \times 10^{5}$ cells $/ \mathrm{ml}$ in $35 \mathrm{~mm}$ diameter petri dishes with $2 \mathrm{ml}$ of medium. This resulted in an average coverage of 260 cells per $\mathrm{mm}^{2}$. Cells that that fall into the glass/poly-1-lysine channels attach and grow. Cells that land on the Cytop do not adhere and float leading to an initially messy image. This can been seen in the floating clumps in Fig. 4(a) Gentle washing for a long period with new medium can remove these floating cells, but it is generally better to only replace half the medium every 3 days. Cell viability studies with FDA showed that cells inside the channels were functioning normally, as can be seen in Fig. 4(c). Floating cells also fluoresced somewhat indicating that their membranes were intact. Fixation and washing for antibody tagging and fluorescent imaging usually removes these floating cells and imaging is not a problem.

\section{Optical and surface properties of the Cytop pattern}

Glass transition temperature of the Cytop is $108^{\circ} \mathrm{C}^{20}$. Multiple uses with a dry oven for annealing and sterilization therefore degrades the Cytop wall angle. The "annealing" of the Cytop does however, return its hydrophobic properties. The hydrophobic quality of the cytop can be seen by the contact angle drops of water make with its surface. The contact angle of clean annealed Cytop is $110^{\circ}$. Cytop also acts as a good electrical insulator. The volume resistivity is greater than $10^{17} \mu \mathrm{cm}$ and has an electric breakdown at $110 \mathrm{KV} / \mathrm{mm}^{19}$. The dielectric constant is 2.1 . Thus it is possible to use Cytop as a dielectric insulator over any electrode structures in the pattern. 
The optical properties of perfluoropolymers are closely related to their structure. Crystalline polymers such as polytetrafluoroethylene (PTFE - Teflon) are opaque. Conversely Cytop is an amorphous polymer and has a transmittance of $95 \%^{20}$ in the range $200-2000 \mathrm{~nm}$. Thus a whole range of optical experiments is possible included those involving UV. The refractive index of Cytop is 1.34 , which is very close to that of water (1.33). The refractive index of glass varies depending on the glass but is typically 1.5-1.6. The similarity in refractive index between Cytop and water means that photolithographic patterns, which are less than $0.5 \_\mathrm{m}$ high, are difficult to image in liquid, even with phase and differential contrast techniques.

There is a tendency for axons and dendrites extending from the neurons to grow along the Cytop walls, especially in the wider channels. This makes imaging of the neurite outgrowths difficult using normal contrast microscopy. The diffraction shadow around the wall obscures imaging of the neurites when using contrast optics. However, fluorescence optics is, not affected in the same way as transmission optics. Stimulation is carried out by scanning a laser from the underside of the neurons, and collection of the fluorescence scattering is also carried out from below. Thus the optical path is not affected by the Cytop wall. Images showing both the topographical structures and the cell features can be obtained by overlaying the fluorescence images with optical contrast images of the pattern structure.

Tau proteins are microtubule associated proteins, which have a molecular weight of 55-65kD. Microtubules function as structural and mobility elements in mitosis, intracellular transport, flagellar movement and in the cytoskeleton. These intracellular filamentous structures are present in the neuron soma, the dendrites and to a lesser extend in the neuron axons ${ }^{21}$. Thus, using Anti-tau antibodies it is possible 
to tag neurite outgrowths, which are sometimes hidden due to the diffraction effects around the Cytop walls. In Fig. 5 a comparison between differential interference contrast and fluorescent images of the tau proteins on the neurons can be seen. In Fig. 5(a) the neurites extending from the soma can be difficult to make out, especially when they are along the walls. The fluorescence image in Fig. 5(b) clearly shows the whole neuron network.

\section{SNOAM imaging}

One of the aims of this research is to allow for the possibility of SPM imaging of the patterned neurons. SPM techniques such as SNOAM offer information not possible with optical techniques alone but also do so at very high resolutions. The limitation with SNOAM and other scanning probe microscopies is that they are surface techniques and depend on scanning a probe across a surface. Thus rough samples can create blind spots and artefacts.

Channel depth will affect topographic patterning of the neurons. The deeper the channels, the harder it is of neurons to expand physically out of them and present a greater barrier for neuron growth. Deep channels however make it difficult for SNOAM imaging. SNOM's based on shear force mode require very flat samples and deep trenches would be very difficult to image. Tapping mode SNOAM can image deep channels and are limited largely by the aspect ratio of the probe and the scan conditions. Compared to AFM, the aspect ratio is poor. This results from the need for high optical throughputs. Low aspect ratio probes can provide higher optical throughput and thus a better signal to noise ratio and optical resolution. High aspect ratio probes conversely provide better topographic resolutions on rough samples, but 
worse optical clarity. Thus the SNOAM probe aspect ratio is a compromise between optical signal and the aspect ratio requirements.

It is possible to image channel walls with angles up to $60^{\circ}$ to the horizontal base with SNOAM probes. Walls steeper than this will have a blind area to the probe. The exact angle possible is dependent also on the feedback conditions. Scans need to be slow enough, and a sufficient feedback loop put in place for the probe to respond accurately to the changing height. In general scan speeds of $7.2 \_\mathrm{m} / \mathrm{s}$ were sufficient for good imaging. Increasing the sample probe distance can also improve the topographic signal, but this worsens the optical signal. On the steeper parts of the walls, the optical scattering increases and the optical transmission signal becomes dark. This can be used to correlate the topographic image to understand the real wall angle. Fluorescent imaging is only affected by the distance of the probe to the sample and is imaged normally.

On flat samples such as in Fig. 6 neurons can be imaged normally. The height of the neuron soma is $700-800 \mathrm{~nm}$, but the neuron topology is not so steep. Thus in this case the imaging limitations are purely to do with the tip size. For SNOAM probes, this is between $300-500 \mathrm{~nm}$ depending on the probe. In deeper channels such as the 3.8_m channel in Fig. 7 there will be a blind spot to the SNOAM probe. For a $60^{\circ}$ imaging capability as described above, a 1_m channel will have a horizontal blindspot of 0.6_m from its base. Likewise the 3.8_m deep channel in Fig. 7 has a blind spot of 2.2_m. However, dendrites are usually of width $0.5-1 \_\mathrm{m}$ and of height $0.2-$ 0.4_m, so it is still possible to image the neurites that grow against the walls, if the wall height is low enough. Generally, the smaller the wall, the better for the SNOAM imaging, but this needs to be balanced with the need for topographic confinement. In 
general, walls of about 1_m tend to be the best compromise between the two. Despite these problems imaging of neurons has been successful in both deep and narrow channels. It even been possible to image a growth cone extending vertically from a 5_m deep channel in Fig. 8.

The dimensions of the neuron soma in the channels vary according to the channel width. Neuron nuclei are in general 10-20_m in widths. In flat areas such as in Fig. 6, or wide channels such as in Fig. 7, the neuron widths are around 10_m. In Fig. 8 and Fig. 9 the horizontal dimensions are distorted by the channel. They are squashed in from the side and extend out along the channel. The exact dimensions are difficult to image in Fig. 8 because of the effect of the vertical neurite extending from the soma. The height of the soma is however noticeably bigger due to their physical confinement affects their dimensions. In the 10_m channel in Fig. 9 the height of the neuron rises to 2_m compared to an average of 1_m or less for neurons on open flat surfaces as seen in Fig. 7. The height of the neuron in the 20_m channel in Fig. 8 is distorted slightly due to the vertical extension of a growth cone.

Initial immuno-fluorescence tagging of NMDA receptors was carried out to show the possibilities of using this system for LTP studies. The cells in this study are not specifically hippocampal neurons, but nevertheless express some NMDA receptors, which can be imaged with immuno fluorescenct tagging.

In Fig. 10, a cell can be seen with a neurite growing along the Cytop wall. The wall side had been exposed to Poly-l-lysine, so growth in this direction was possible. In this case the aspect ratio of the wall was reduced to much less than $90^{\circ}$ due to repeated heating during the recycling process. What can be seen is that even neurites along the wall can be imaged with this technique due to the size of neurite relative to 
the wall. Fluorescence imaging shows fluorescence around the main cell soma and around a suspected synaptic area. The dendrite shows residual fluorescence, but this is much less than that shown in this area.

\section{DISCUSSION}

In this paper we have presented a system of patterning primary culture neurons that can guide and isolate individual neurite growth. In addition we have proved that it is possible to do SPM imaging in our 3 dimensional surface structures. This method is compatible with other cell lines such as $\mathrm{PC} 12^{8}$ and $\mathrm{SH}-\mathrm{SY} 5 \mathrm{Y}^{22}$, and to SPM analysis. This research is the initial step for an ongoing project to pattern neurons, electrically stimulate and record from them and to analyze them with fluorescence microscopy and SPM. To this end the transparent and open top nature of this structure is significant because it will provide the experimental biologist with a new growth substrate that can control the placement and growth of neurons while allowing fluorescent imaging.

The addition of not only SPM analysis, but also perhaps SPM manipulation will provide the basis for future experiments in this area. Using apertured SNOAM probes in conjunction with, for example, caged molecules could provide a method for ultra localized delivery of biochemicals. The physical localized forces from probe tips can also be used for manipulations involving pressure and cutting.

Patterning has been shown to be successful with Poly-L-lysine. In some cases neuroscientists prefer not to use poly-l-lysine due to the effect of the positively charged amide groups on the neuron physiology and function. This is not a problem in our system since it is also possible to put collagen or other growth matrixes through 
the microfluidic patterning system. The growth system described above is thus quite flexible to the needs of different cells.

\section{ACKNOWLEDGEMENTS}

We thank the JSPS, CNRS, the Japan Society for the Promotion of Science, French Embassy (service of Science and Technology), the local government of Brittany, and the Monbusho scholarship program for funding this work. 


\section{REFERENCES}

[1] Bliss T.V.P., Collinbridge G.L. (1993) A synaptic model of memory: Long-term potentiation in the hippocampus Nature 361, 31-39

[2] Ehlers M.D., Mammen A.L., Lau L, Hunganir L. Synaptic targeting of glutamate receptors (1996) Current Opinion in Cell Biology 8, 484-489

[3] Petralia R., Yokotani N., WentholdR. (1994) Light and electron microscope distribution of NMDA receptor subunit NMDAR1 in the rat nervous system using a selective anti-peptide antibody J. Neurosci. 14(12) 667-696

[4] Tamiya E., Nagatami N. Iwanuchi S., Murakami Y., Sakaguchi T., Yokoyama K. (1997) Simultaneous topographic and fluorescence imagings of recombinant bacterial cells containing a green fluorescent protein gene detected by a scanning near-field optical/atomic force microscope Anal. Chem. 69, 36973701 .

[5] Iwabuchi S., Muramatsu H., Yamamoto N., Murakami Y., Yokoyama Y., Tamiya E. (1996) Scanning Near-field optical/atomic force microscopy for fluorescence imaging and spectroscopy of biomaterials in air and liquid: Observation of recombinant Escherichia coli with gene coding to green fluorescent protein. Optical Review 3, 470-474

[6] Degenaar P. Murakami Y, Yokoyama K, Tamiya. E. (2000) Near field imaging of NMDA receptors on patterned neuron networks, Proc. NFO-6, 271

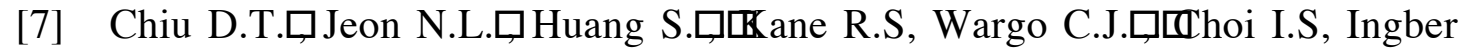
D.E. $\square$ Whitesides G.M. $\square(2000)$ Patterned deposition of cells and proteins onto surfaces by using three-dimensional microfluidic systems PNAS 97, 2408-2413.

[8] Matsuda T.प्Sugawara T.प्Inoue K.ㅁ1992)Two-dimensional cell manipulation technology an artificial neural circuit based on surface microphotoprocessing, ASAIO Journal 243-247.

[9] Mrksich M., Dike E.L., Tien J., Ingber D.E., Whitesides G.M. (1997) Using Microcontact printing to pattern the attachment of mammalian cells to SelfAssembled Monolayers of Alkanethiolates on Transparent Films of Gold and Silver. Exp. Cell. Res.235, 305-313

[10] Martinoia S., Bove M., Tedesco M., Margesin B., Grattarola M. (1999) A simple system for patterning populations of neurons on silicon micromachined substrates, J. Neurosci. Methods 87,35-44

[11] Degenaar P. Griscom L., Le Pfioufle B., Murakami Y., Yokoyama K. Fujita H., Tamiya E. (2001), Microfluidic chips for screening of neuron drugs, Proc. Biochips 2001 
[12] Giovangrandi L.(1999) Biopatterning of neural cells on microelectrode arrays. Thesis, École Polytechnique Fédérale de Lausanne, Lausanne, Switzerland

[13] Shirakawa T., Honma S., Katsuno Y., Oguchi H., Honma K. (2000) Synchronization of circadian firing rhythms in cultured suprachiasmatic neurons. Eur. J. Neurosci. 12, 2833-2838

[14] Makohliso S.A., Giovangrandi L., Léonard D., Mathieu H.D., Ileghems M., Aebisher P. (1998) Application of Cytop-AF thin films for biopatterning of neural cell adhesion, Biosensors and Bioelectronics 13(11),1227-1235

[15] Griscom L., Degenaar P., Le Pfioufle B., Tamiya E. Fujita (2001) Soft Lithographic Techniques For Guidance Of Hippocampal Neurons On MicroElectrode Arrays Proc Transducers 2001

[16] Muramatsu H., Ataka T., Chiba N., Monobe H., Fujihira M. (1995) Scanning near-field optic/atomic microscopy Ultramicroscopy 57, 141-146

[17] Corey J. M., Wheeler B. C., Brewer G. J. (1996) Micrometer Resolution of Silane-Based Patterning of Hippocampal Neurons $\square$ Critical Variables in Photoresist and Laser Ablation Processess for Substrate Fabrication IEEE transactions on Biomedical Engineering 43(9),944-955

[18] Kunz S., Spring M., Ginsburg C., Buchstaller A., Berger P., Lanz R., Rader C., Vogt L., Kunz B., Sonderegger P. (1998) Neurite fasciculation mediated by complexes of axonon-1 and $\mathrm{Ng}$ cell adhesion molecule, J. Cell Biol. 143(6), $1673-1690$.

[19] Bellex International, Cytop data sheet, Bellex international home page, http://www.bellexinternational.com

[20] Asahi Glass Company, Cytop data sheet, Asahi Glass company home page home page http://www.agc.co.jp

[21] Mandell J.W., Banker G.A. (1996) A spatial gradiaent of tau protein phosphorylation in nascent axons $J$. Neurosci. 16, 5727-5740

[22] Uberti D., Rizzini C., Spano P.F., Memo M. (1997) Characterization of tau proteins in human neuroblastoma SH-SY5Y cell line. Neuroscience Letters 235(3), 149-53 
(a) Cytop pattern fabrication

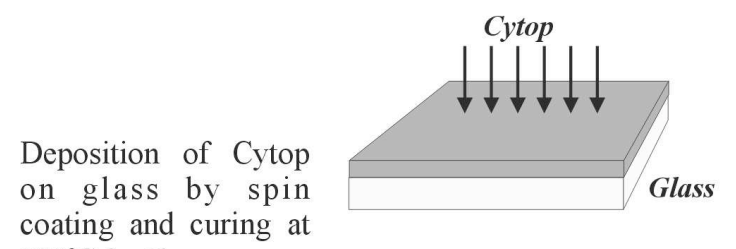

$200^{\circ} \mathrm{C}$ for lhour.

Deposition of $200 \mathrm{~nm}$ of Al by evaporation.

Patterning of the Al by lithography and Chemical etching.

$\mathrm{Al}$ is used as a mask to etch Cytop by ashing with RIE.

Removal of Al using Al chemical etchant. Then cleaning.

\section{(c) Alignment and} matrix deposition
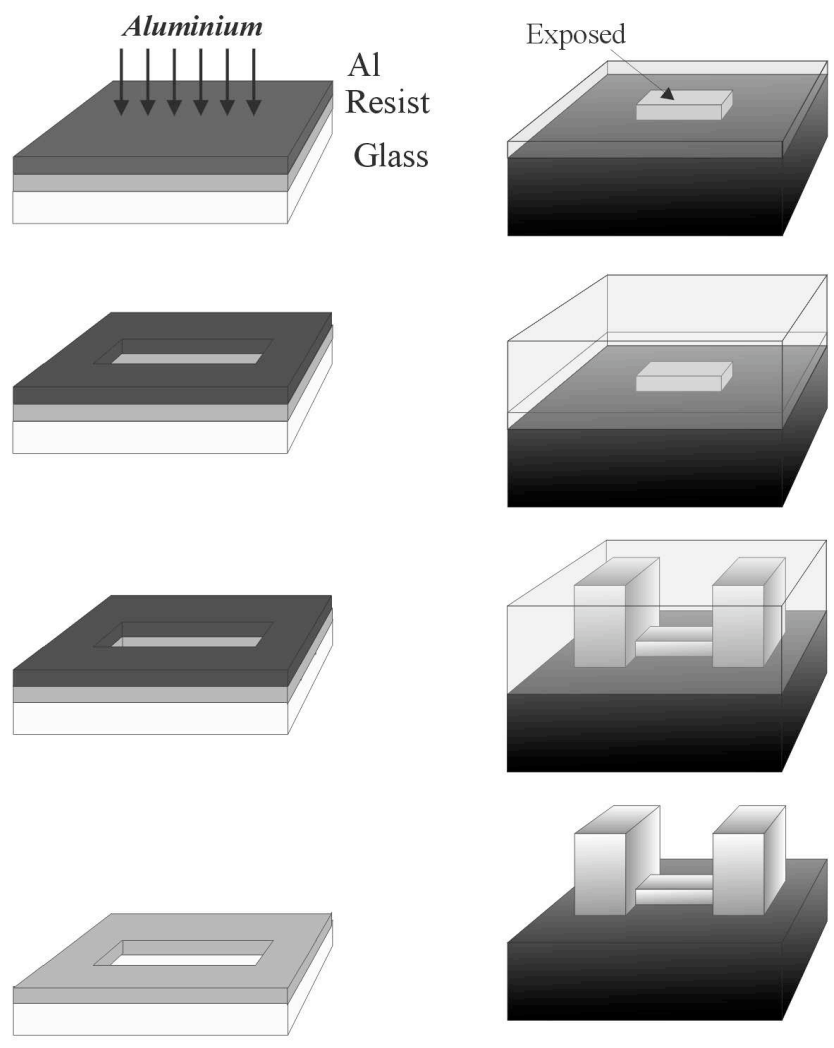

2nd spin coat $100 \mu \mathrm{m}$ thick

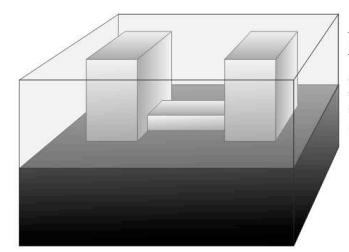

Exposure using mask 2 followed by baking

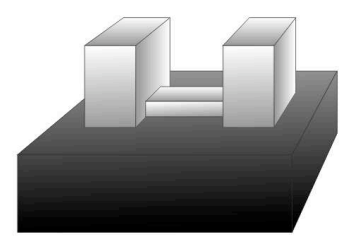

Develop

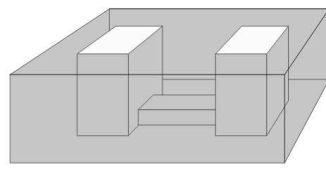

Mold P D S stencils. Expose stencils to oxygen plasma for $1 \mathrm{~min}$ to make hydrophilic

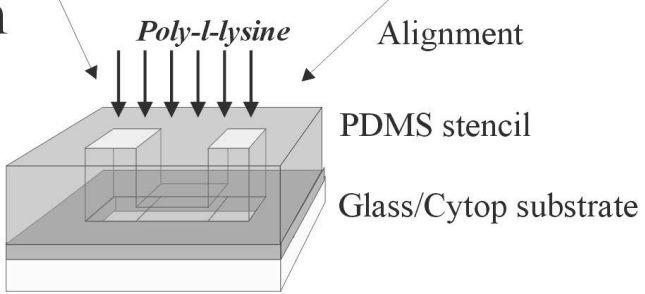

ehydrophlc

Fig. 1. Fabrication of the Cytop/poly-l-lysine structures on glass substrates. Initially Cytop structures are made on the glass substrate. Then a PDMS stencil is made using an SU-8 molding process. Finally the PDMS stencil is aligned to the glass/Cytop substrate and a poly-lysine coat is formed through immersion in poly-lysine solution 


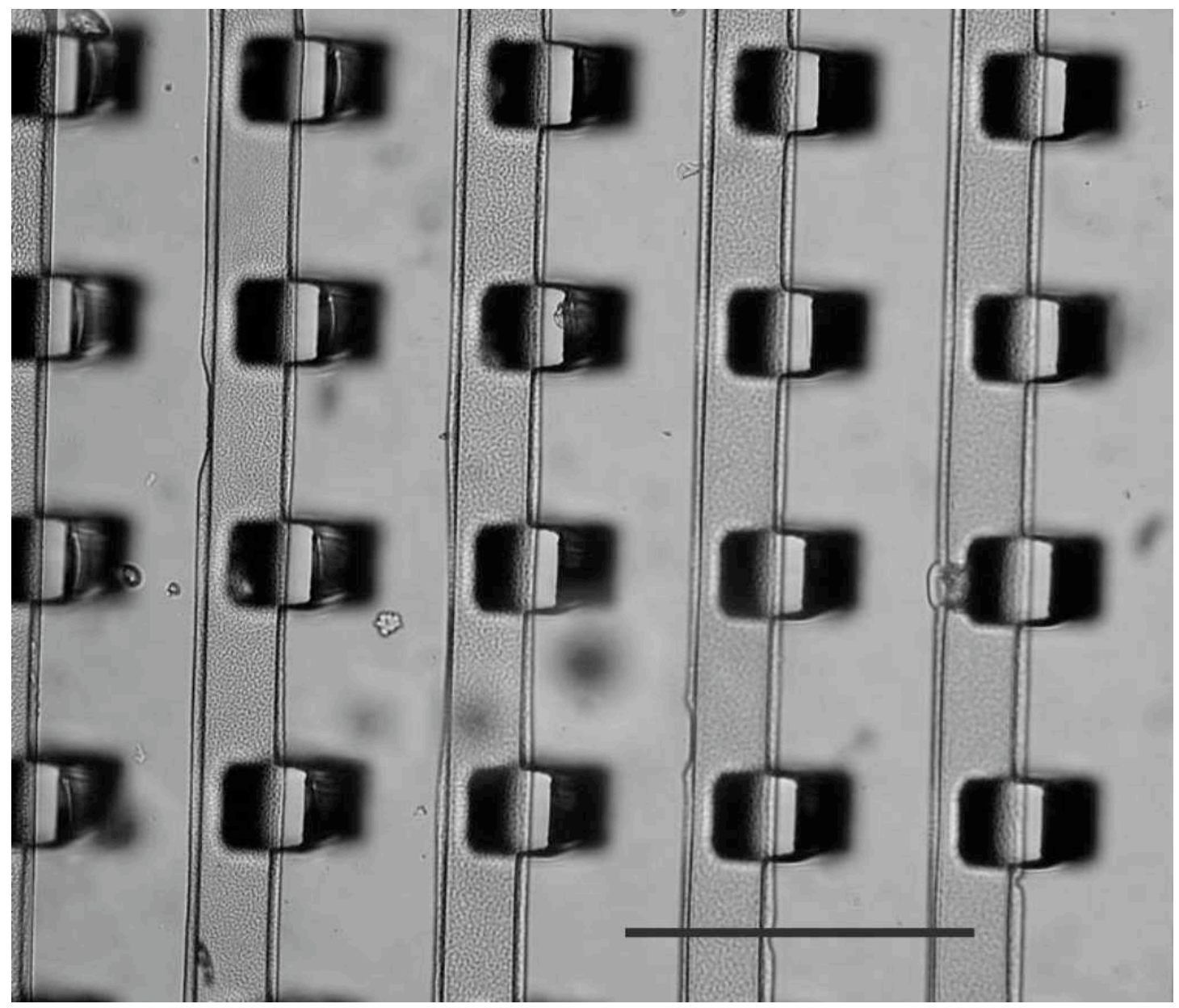

Fig. 2. Alignment of the PDMS to the Cytop strips. In this case a 50micron wide channel PDMS microfluidic system was aligned to a 50_m wide lined Cytop/glass pattern. With normal optical microscopes, an alignment precision of 1 or 2 _m is possible. Scale bar is 200_m 

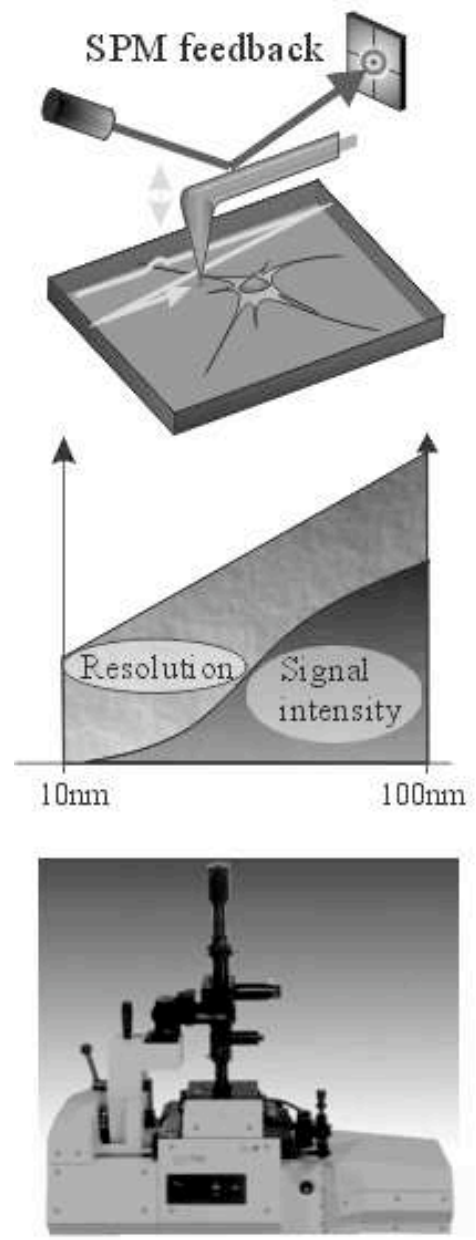

\section{SII SPA700 SNOAM}

Fig. 3. The scanning near-field optical atomic force microscope. Samples are scanned underneath a vibrating apertured probe to build up simultaneous topographic and near-field optical images. Atomic force feedback keeps the probe several nanometres from the surface and the dipole-dipole interaction between the evanescent field at the probe tip and the sample gives diffraction beating optical resolutions. 


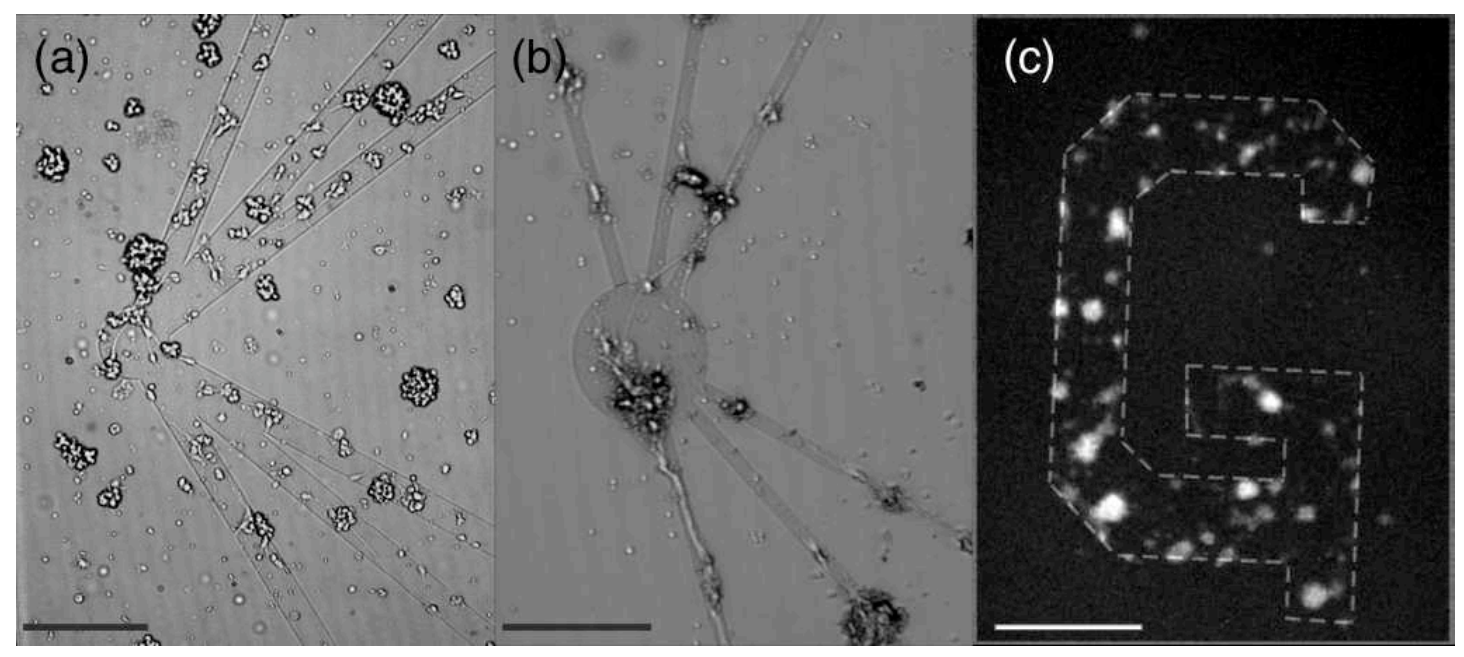

Fig. 4: Optical contrast and fluorescent images of patterned neurons. (a) shows cells still in the live state some clouds of neurons which did not adhere to the surface can be seen. Scale bar is 200_m (b) shows neurite growths on 10_m wide paths. At this scale patterning of individual neurites can be achieved. Scale bar is $100 \mathrm{~m}$. (C) shows a fluorescent image taken with fluorescein diacetate. The pattern in this case is the shape of a $\mathrm{G}$ and the scale bar is 100_m. 


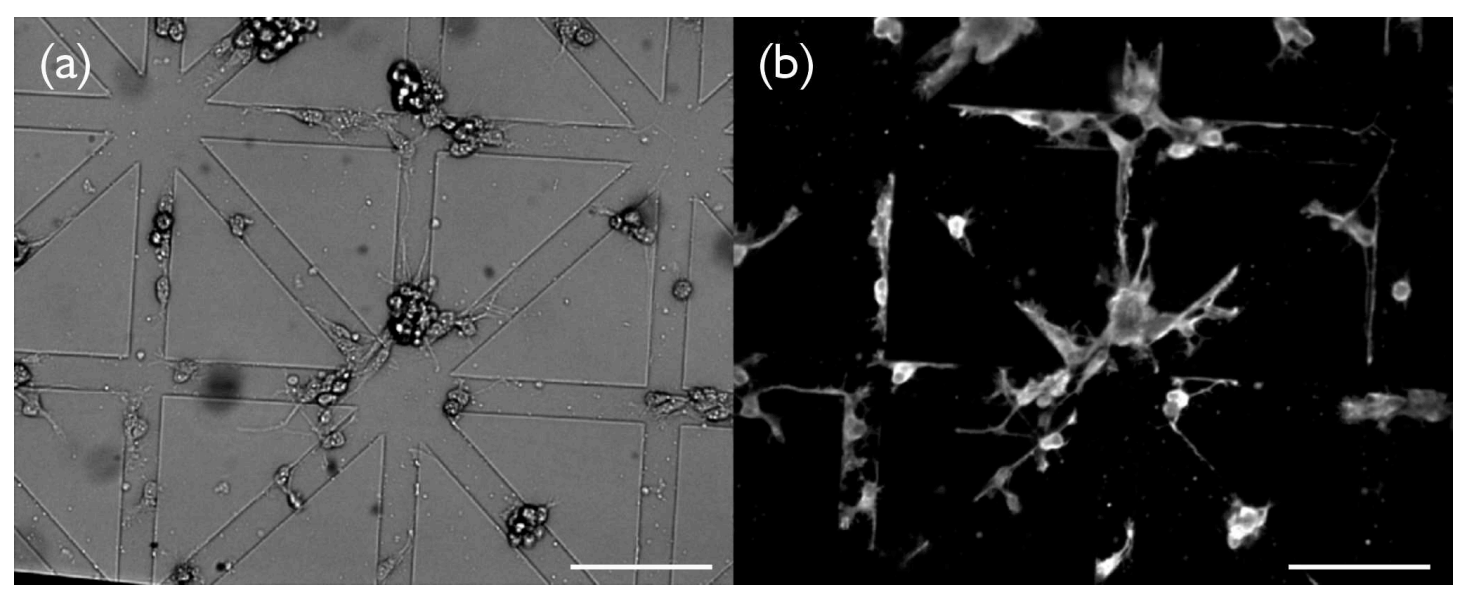

Fig. 5. Differential interference contrast (a) and fluorescence (b) micrographs of neurons growing in the Cytop patterns. Neurons have been grown for 3 days and immuno tagged with anti-tau antibodies. A slight amount of unspecific growth is occurring to the south of the central trench, and is probably due to a slight misalignment of the PDMS stencil in this case. The scale bar is 200_m 

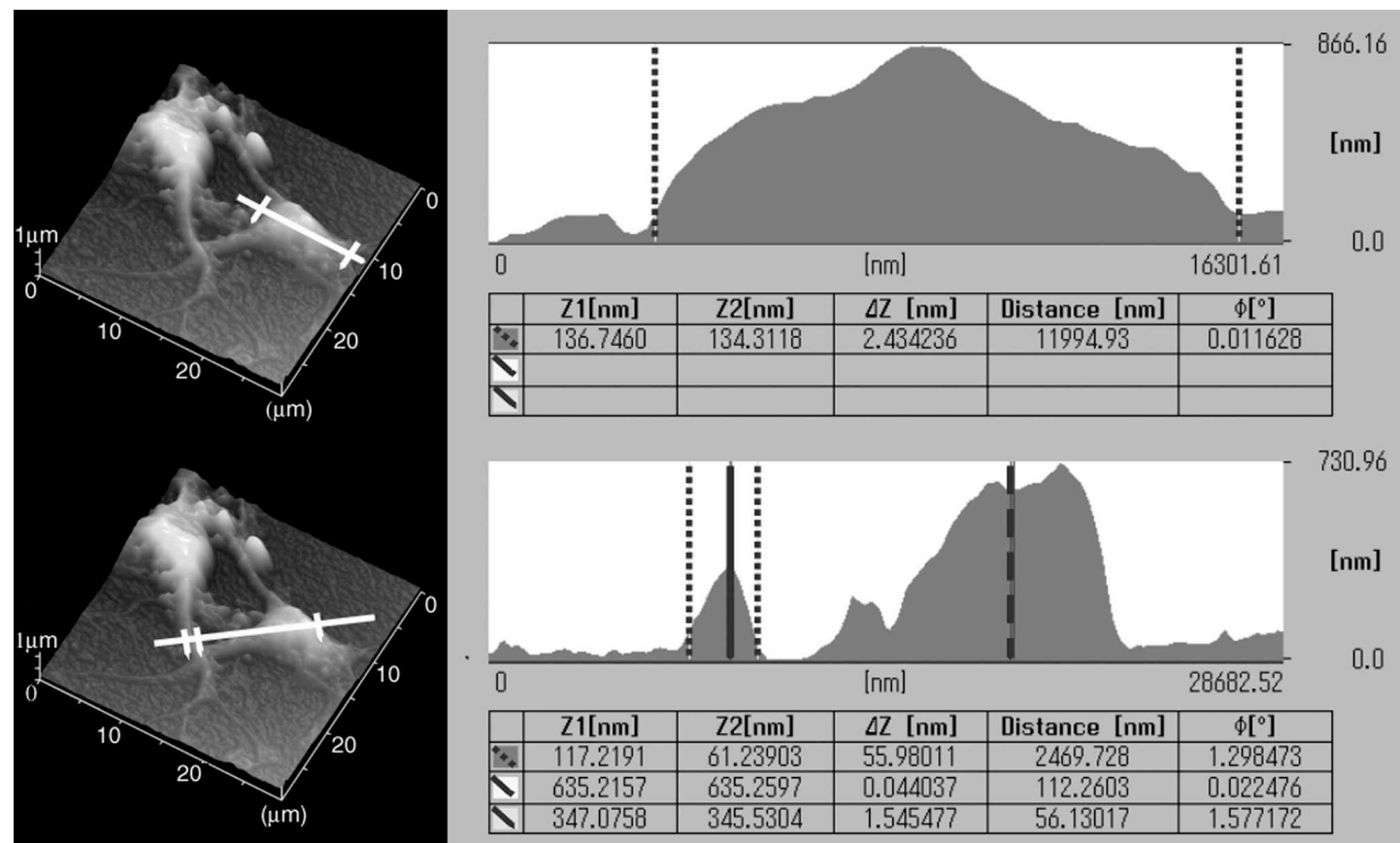

Fig. 6. Neurons on an open flat glass substrate of scan area $72 \_\mathrm{m}^{2}$. In situation the height of the neuron cells is less than 1_m. The height of dendrites is measured as $350 \mathrm{~nm}$. 


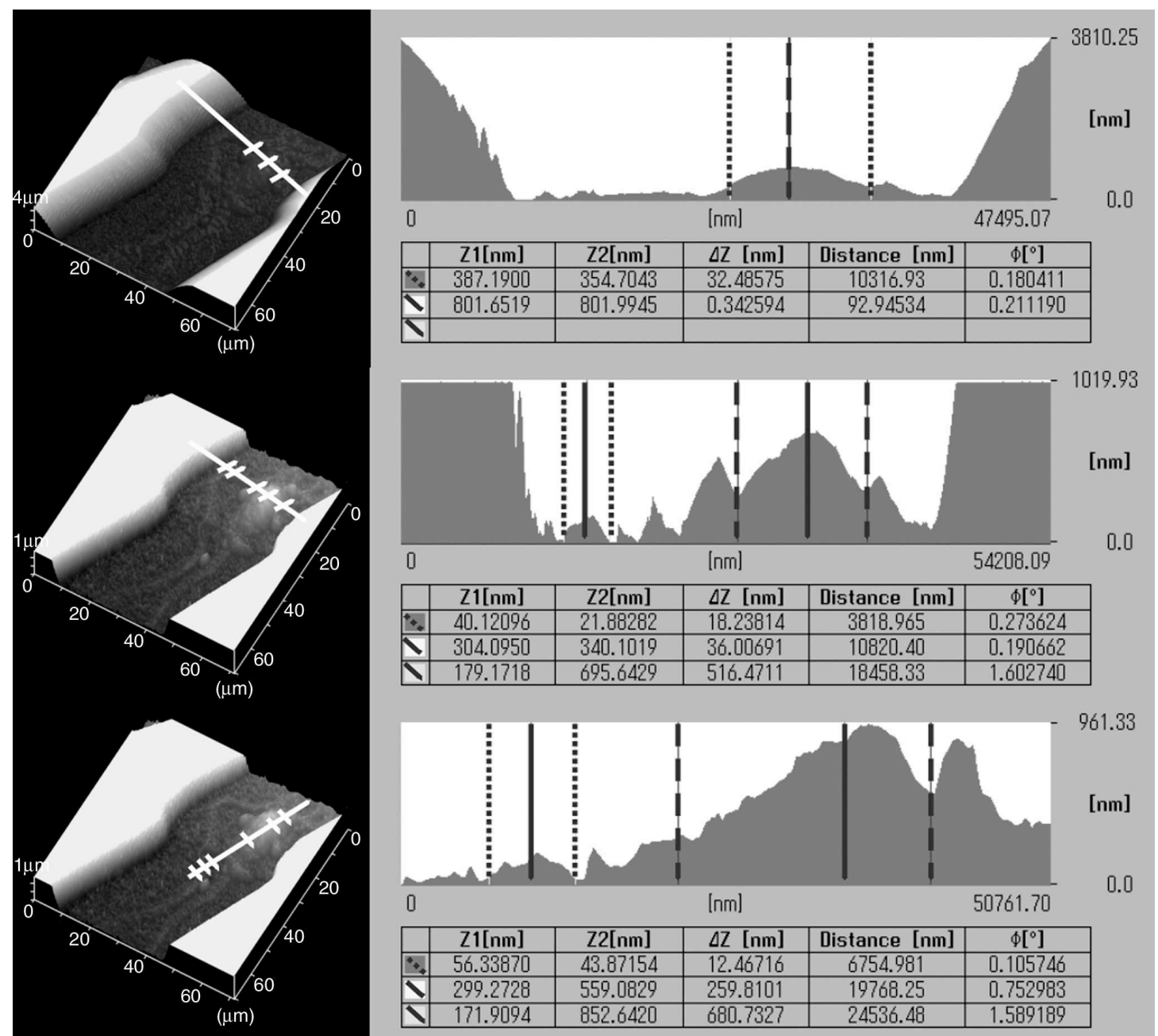

Fig. 7. Neurons inside a 30_m wide channel. The channel height is 3.8_m. The top image is the original profile, while the middle and bottom images have had the profile digitally clipped at 1_m to show better contrast. The some and neurites growing inside the channel are clearly visible. Neuron height is just under 1_m and the dendrite height is just under 200nm. 


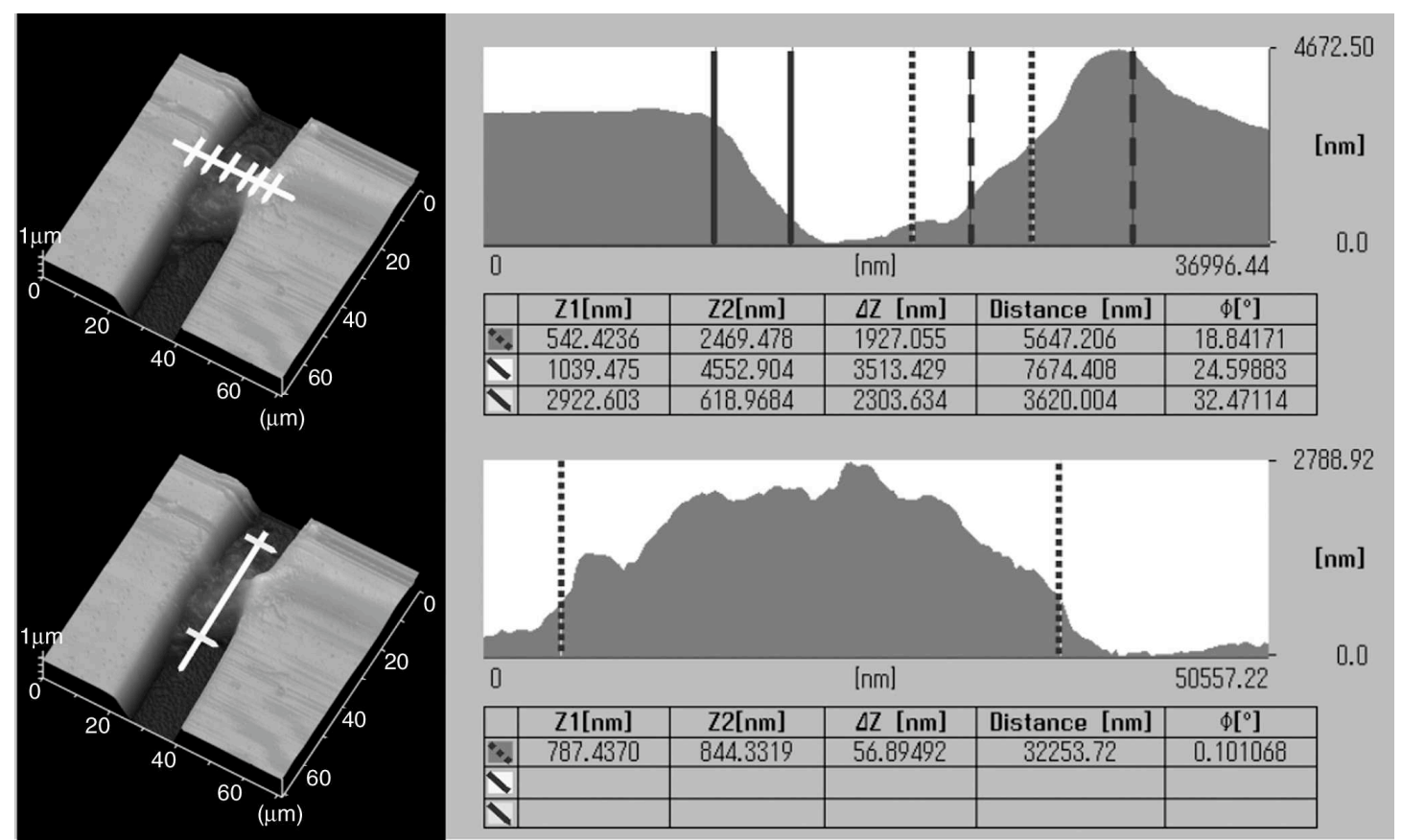

Fig. 8. Neurons inside a 20micron wide channel. Channel depth is 4.5 m. In this case a growth cone is pushing up vertically from the cell and has stopped when it reached the Cytop surface layer. This vertical neurite is responsible for the distortion in the channel profile in the top image. In the bottom image the neuron height is 2.8 m as a result of compression between the walls. 

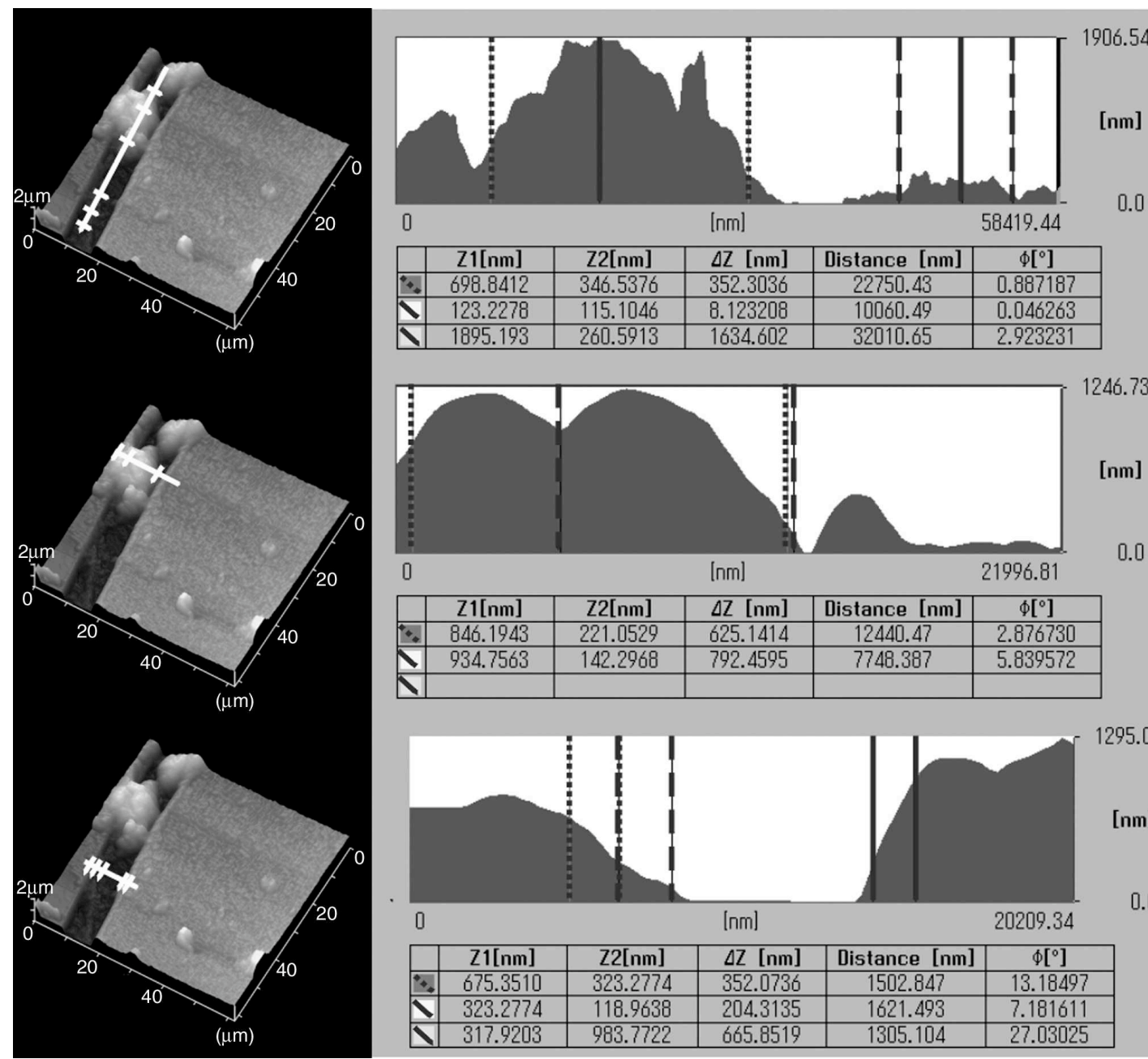

246.73

$[\mathrm{nm}]$

0.0

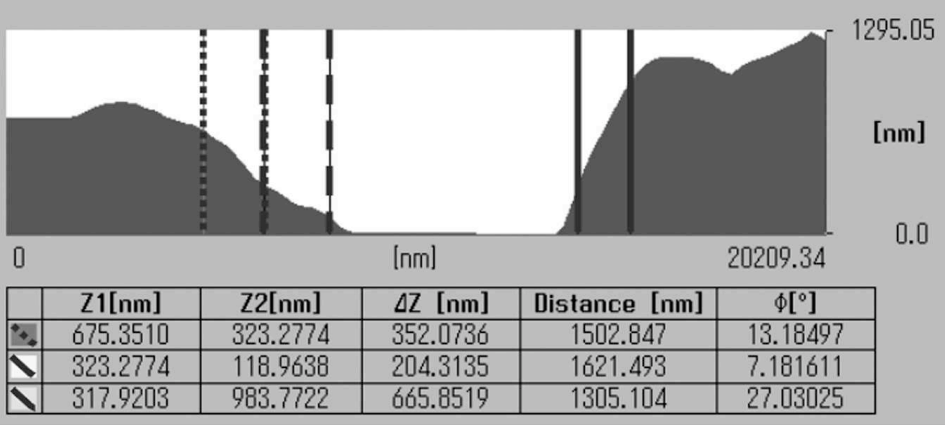

Fig. 9. Neurons inside a 10_m channel. In this case the channel height is 1_m. At this height it is easier for the cells to push over the top, and spread out over the Cytop surface (Bottom image). The height of the cell in this case is just under 2_m. This is lower than for the 20_m channel because the cell has been able to spread over the 1_m wall 


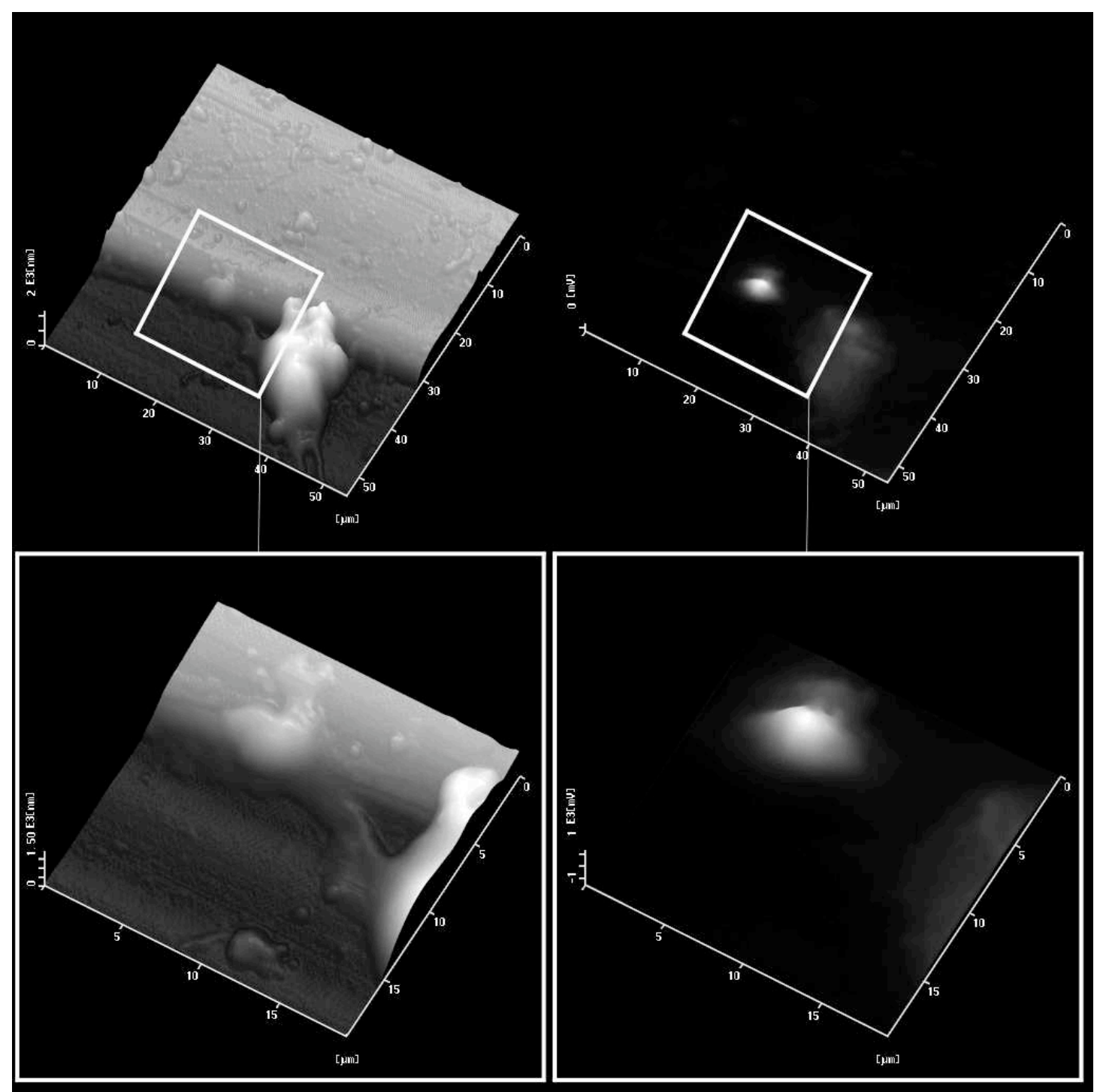

Fig. 10. Topographic (Left) and fluorescent (Right) SNOAM images of NMDA receptors on a neuron growing inside a wide channel. In this case the wall angle is much less than $90^{\circ}$ due to multiple recycles. A neurite growing from the Neuron soma can clearly be seen to be growing along the wall. Fluorescence occurs at the soma and at the site of a probable synapse. 